\title{
Comparative genomic analysis of Yersinia pestis strains of the philogenetic branch 2.MED4
}

\author{
A.N. Balykova \\ Russian Research Anti-Plague Institute \\ "Microbe", Federal Service for Surveil- \\ lance in the Sphere of Consumers \\ Rights Protection and Human Welfare \\ Saratov, Russia \\ alinabalnik@gmail.com
}

\author{
Zh.V. Al'khova \\ Russian Research Anti-Plague Institute \\ "Microbe", Federal Service for Surveil- \\ lance in the Sphere of Consumers \\ Rights Protection and Human Welfare \\ Saratov, Russia
}

\author{
G.A. Eroshenko \\ Russian Research Anti-Plague Institute \\ "Microbe", Federal Service for Surveil- \\ lance in the Sphere of Consumers \\ Rights Protection and Human Welfare \\ Saratov, Russia
}

\begin{abstract}
Keywords - Yersinia pestis, strains of medieval biovar, comparative SNP-analysis, SNP-genotyping, phylogenetic analysis
\end{abstract}

\section{Motivation and Aim}

Highly virulent gram-negative bacterium Yersinia pestis, the causative agent of plague, is one of the most outstanding example of pathogens that caused huge damage to the health of mankind. Our molecular investigations showed that strains of medieval biovar (phylogenetic branch 2.MED) are the most common in the natural foci of plague with different geographical landscapes and occupy about $80 \%$ of the territory of the natural foci of CIS [1].

In the 18th-19th centuries, numerous outbreaks of plague with high mortality rates were registered in the Caspian-Sea region, Russia. Our research prove that since 1912, outbreaks in the Northern Caspian-Sea region were caused by strains of medieval biovar. We identified 3 Y. pestis strains, which were isolated in this region and formed the previously unidentified phylogenetic branch 2.MED4. The strains of branch 2.MED4 have high virulence and epidemic significance and can cause plague in animals and humans $[2,3]$.

This information determines the importance of further research of 2.MED4 strains. In this regard, it is important to establish their place in the evolutionary scheme of medieval biovar, to determine their role in plague outbreaks in the Caspian-Sea region, and to assess the possibility of their return to these territories. The aim of this study was to identify the specific molecular-genetic features of $Y$. pestis strains of the phylogenetic branch 2.MED4 and to develop a method for their identification by PCR.

\section{Methods and Algorithms}

High-performance sequencing was carried out using Ion PGM system (Life technologies). Ion Torrent Suite software 3.4.2 and Newbler gsAssembler 2.6 were applied for data processing. For compara-tive SNP-analysis and phylogenetic reconstruction bioinformatics programs (Wombac 2.0, jModelTest, PAUP 4.0, PhyML 3.1, MEGA X, FigTree 1.4.3.) were used. Fragment sequencing was performed using the ABI PRISM 3500XL platform (Applied Biosystems, USA).

\section{Results}

We performed comparative SNP-analysis of $3 Y$. pestis stains of branch 2.MED4. We found 10 SNPs, which specific to this branch and developed a method for SNP-genotyping of these strains by PCR. We explored $26 Y$. pestis strains from the plague foci of the Caspian-Sea region by PCR and found and found 5 more $Y$. pestis strains of branch 2.MED4.

We have sequenced and carried out complex analysis of molecular-genetic and phenotypic properties of $5 Y$. pestis strains of the previously unidentified phylogenetic branch 2.MED4.

According to the complex analysis of phenotypic and genetic properties, all strains are typical of medieval biovar of $Y$. pestis. According to PCR-/SNP-genotyping and phylogenetic analysis, all of $5 Y$. pestis strains belong to phylogenetic branch 2.MED4, as well as 3 previously identified strains.

Our results may be useful for a retrospective analysis of plague outbreaks and for identifying patterns of evolution of medieval biovar in the Caspian-Sea region in the 18th-20th centuries.

\section{Conclusion}

We have sequenced and analyzed $8 Y$. pestis strains of the previously unidentified phylogenetic branch 2.MED4. We found 10 marker SNPs, specific to this branch and developed a method for SNP-genotyping of these strains by PCR. The data obtained can be used to improve the methods of molecular-genetic identification of $Y$. pestis and to determine patterns of evolution and distribution of medieval biovar in the natural plague foci of the Caspian-Sea region.

\section{REFERENCES}

[1] Kutyrev V. V. et al. (2018). Phylogeny and classification of Yersinia pestis through the lens of strains from the plague foci of Commonwealth of Independent States. Front. Microbiol. 9:1106. DOI: 10.3389/fmicb.2018.01106

[2] Eroshenko G. A. et al. (2019). Circulation of Yersinia pestis in the Volga-Ural Sandy fo-cus: spatiotemporal analysis. Problems of Particularly Dangerous Infections. 2019;3:51-57. DOI: 10.21055/0370-1069-2019-3-51-57.

[3] Eroshenko G.A., Popov N.V., Al'khova Zh.V., Balykova A.N., Kukleva L.M., Kutyrev V.V. (2019). Phylogenetic analysis of Yersinia pestis strains of medieval biovar, isolated in Pre-caspian NorthWestern Steppe plague focus in the XX century. Problems of Particularly Danger-ous Infections. 2019; 2:55-61. DOI: 10.21055/0370-1069-2019-2-55-61. 